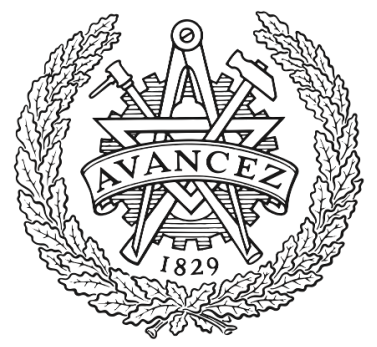

CHALMERS

UNIVERSITY OF TECHNOLOGY

\title{
Electrochemical Model-Based Fast Charging: Physical Constraint-Triggered PI Control
}

Downloaded from: https://research.chalmers.se, 2023-04-26 11:54 UTC

Citation for the original published paper (version of record):

Li, Y., Vilathgamuwa, D., Wikner, E. et al (2021). Electrochemical Model-Based Fast Charging: Physical Constraint-Triggered PI Control. IEEE Transactions on Energy Conversion, 36(4): 3208-3220. http://dx.doi.org/10.1109/TEC.2021.3065983

N.B. When citing this work, cite the original published paper.

C2021 IEEE. Personal use of this material is permitted.

However, permission to reprint/republish this material for advertising or promotional purposes 


\title{
Electrochemical Model-Based Fast Charging: Physical Constraint-Triggered PI Control
}

\author{
Yang Li, Member, IEEE, D. Mahinda Vilathgamuwa, Fellow, IEEE, Evelina Wikner, Member, IEEE, \\ Zhongbao Wei, Member, IEEE, Xinan Zhang, Member, IEEE, Torbjörn Thiringer, Senior Member, IEEE, \\ Torsten Wik, and Changfu Zou, Member, IEEE
}

\begin{abstract}
This paper proposes a new fast charging strategy for lithium-ion (Li-ion) batteries. The approach relies on an experimentally validated high-fidelity model describing battery electrochemical and thermal dynamics that determine the fast charging capability. Such a high-dimensional nonlinear dynamic model can be intractable to compute in real-time if it is fused with the extended Kalman filter or the unscented Kalman filter that is commonly used in the community of battery management. To significantly save computational efforts and achieve rapid convergence, the ensemble transform Kalman filter (ETKF) is selected and tailored to estimate the nonuniform Li-ion battery states. Then, a health- and safety-aware charging protocol is proposed based on successively applied proportional-integral (PI) control actions. The controller regulates charging rates using online battery state information and the imposed constraints, in which each PI control action automatically comes into play when its corresponding constraint is triggered. The proposed physical constraint-triggered PI charging control strategy with the ETKF is evaluated and compared with several prevalent alternatives. It shows that the derived controller can achieve close to the optimal solution in terms of charging time and trajectory, as determined by a nonlinear model predictive controller, but at a drastically reduced computational cost.
\end{abstract}

Index Terms-Electrochemical model, ensemble transform Kalman filter (ETKF), fast charging, lithium plating, lithiumion (Li-ion) battery.

\section{NOMENCLATURE}

Symbol:

$C_{T} \quad$ Battery thermal capacitance $(\mathrm{J} / \mathrm{K})$.

$H \quad$ Prediction horizon of model predictive control.

$I_{I} \quad$ Current due to molar flux of intercalation (A).

$I_{\text {app }} \quad$ Applied charging current (A).

$K_{P}, K_{I}$ Proportional and integral gains.

$M \quad$ Order of the submodel for solid-phase diffusion.

$N^{j} \quad$ Number of control volumes of a domain. $j \in$ $\{$ pos, sep, neg $\}$.

This work was supported by EU-funded Marie Skłodowska-Curie Actions Individual Fellowships under Grant 895337-BatCon-H2020-MSCA-IF-2019.

Y. Li, E. Wikner, T. Thiringer, T. Wik, and C. Zou are with the Department of Electrical Engineering, Chalmers University of Technology, 41296 Gothenburg, Sweden (e-mail: yangli@ieee.org; evelina.wikner@chalmers.se; torbjorn.thiringer@chalmers.se; tw@ chalmers.se; changfu@chalmers.se).

D. M. Vilathgamuwa is with the School of Electrical Engineering and Robotics, Queensland University of Technology, Brisbane, QLD 4001, Australia (e-mail: mahinda.vilathgamuwa@qut.edu.au).

Z. Wei is with the School of Mechanical Engineering, Beijing Institute of Technology, Beijing 100081, China (e-mail: weizb@bit.edu.cn).

$\mathrm{X}$. Zhang is with the School of Electrical, Electronic and Computer Engineering, University of Western Australia, Perth, WA 6009, Australia (email: xinan.zhang@uwa.edu.au).
$N^{\text {tot }}=N^{\text {pos }}+N^{\text {sep }}+N^{\text {neg }}$. Total number of control volumes.

$P_{\text {bat }} \quad$ Battery charging power $(\mathrm{W})$.

$Q_{T} \quad$ Heat generation (W).

$R_{T} \quad$ Battery thermal resistance $(\mathrm{K} / \mathrm{W})$.

$R_{\text {col }} \quad$ Current collector resistance $(\Omega)$.

$R_{\mathrm{ct}} \quad$ Charge-transfer resistance $(\Omega)$.

$R_{e}, R_{s}$ Electrolyte and solid-phase resistances $(\Omega)$.

$R_{\text {SEI }} \quad$ Solid-electrolyte interphase film resistance $(\Omega)$

$\mathcal{S}^{j} \quad$ Set of control volume indices. $j \in\{$ pos, sep, neg $\}$.

SOC State of charge.

$T \quad$ Battery temperature $(\mathrm{K})$.

$T_{\text {amb }} \quad$ Ambient temperature (K).

$U_{\mathrm{sr}}^{\mathrm{ref}} \quad$ Reference voltage of the side reactions (V).

$U_{\text {ss }} \quad$ Equilibrium potential of an electrode $(\mathrm{V})$.

$V_{\text {bat }} \quad$ Battery terminal voltage (V).

$V_{e} \quad$ Electrolyte diffusion overpotential (V).

$\Phi_{e}, \Phi_{s}$ Electrolyte and solid-phase potentials (V).

$c_{e}, c_{s}$ Electrolyte and solid-phase concentrations $\left(\mathrm{mol} / \mathrm{m}^{3}\right)$.

$c_{s, \text { avg }}$ Volume-averaged concentration in the solid phase $\left(\mathrm{mol} / \mathrm{m}^{3}\right)$.

$c_{s, \max }$ Maximum concentration in the solid phase $\left(\mathrm{mol} / \mathrm{m}^{3}\right)$.

$c_{\mathrm{ss}} \quad$ Surface concentration in the solid phase $\left(\mathrm{mol} / \mathrm{m}^{3}\right)$.

e Feedback error.

$j_{I} \quad$ Molar flux due to intercalation $\left[\mathrm{mol} /\left(\mathrm{m}^{2} \cdot \mathrm{s}\right)\right]$.

$m \quad$ Ensemble size.

$n \quad$ System order.

$q \quad$ Output of the integral control.

$\eta_{\mathrm{ct}} \quad$ Charge-transfer (intercalation) overpotential (V).

$\eta_{\text {sr }} \quad$ Side-reaction potential. $s r \in\{\mathrm{LiP}, \mathrm{SEI}\}(\mathrm{V})$.

$\eta_{\text {sr,min }} \quad$ Minimum potential to restrain side reactions. $s r \in$ $\{\mathrm{LiP}, \mathrm{SEI}\}(\mathrm{V})$.

$\Delta t \quad$ Sampling time (s).

$\Delta c_{s} \quad$ Concentration difference term in the solid-phase $\left(\mathrm{mol} / \mathrm{m}^{3}\right)$.

Subscript:

$N \quad$ Control volume at electrode/separator boundary.

$i \quad$ Control volume index. $i \in\left\{1,2, \cdots, N^{\text {tot }}\right\}$.

$i \pm 0.5$ Variable at the edges of $i$ th control volume. $i \in$ $\left\{1,2, \cdots, N^{\text {tot }}\right\}$.

$k \quad$ Discrete time index. $k \in\{0,1, \cdots\}$.

$l \quad$ Index of concentration difference terms in the solid phase. $l \in\{2, \cdots, M\}$. 
$p \quad$ Ensemble member index. $p \in\{1,2, \cdots, m\}$.

$q \quad$ State variable index. $q \in\{1,2, \cdots, n\}$.

$\lambda \quad$ Charging mode. $\lambda \in\{1,2, \cdots\}$.

$0 \quad$ Initial value.

1 Control volume at electrode/current collector boundary.

$0 \% \quad$ Value at $\mathrm{SOC}=0 \%$

$100 \%$ Value at SOC $=100 \%$.

LiP Lithium plating.

SEI Solid-electrolyte interphase film.

\section{Superscript:}

neg Negative electrode.

pos Positive electrode.

sep Separator.

$\top \quad$ Transpose.

$+\quad$ Posterior estimate.

- $\quad$ Prior estimate.

\section{INTRODUCTION}

$\mathbf{T}$ HANKS to its distinct advantages, including the high power and energy densities, low self-discharge rate, favorable modularity, and rapidly declined cost in recent years, lithium-ion (Li-ion) battery has become the major energy storage technology to achieve transportation electrification [1]. In contrast to the fast refueling of conventional fossil-fueled vehicles, Li-ion battery-powered electric vehicles (EVs) suffer from the issue of long charging time. However, simply increasing the battery charging current rate can unfavorably speed up the aging, damage the batteries, and even pose serious hazards to the vehicle users. In this regard, conservative charging control strategies are commonly applied in practice, leading to a significant part of battery capacity being underutilized. The design of a safe, healthy, and efficient strategy for Li-ion battery fast charging is thus highly desirable and has gained a growing interest over the past decade [2], [3].

The existing battery charging strategies can be generally divided into model-free and model-based methods. The modelfree strategies include constant-current constant-voltage (CC$\mathrm{CV}$ ), constant-power CV (CP-CV), CV-CCCV [4], multistage $\mathrm{CC}$ (MCC) [5], and pulse charging [6]. These approaches can be viewed as heuristic or passive methods characterized by predefined profiles with constant current, voltage, and/or power, while the internal dynamics of the battery are completely ignored due to the lack of relevant information. The resultant charging protocols are easy to implement but can be accompanied with long charging time, rapid battery degradation, and unaddressed safety issues.

In contrast, the model-based strategies adopt various dynamic models of the batteries to design charging algorithms. Conventionally, lumped-parameter equivalent circuit models (ECMs) with simple circuit structures [7] or electro-thermal coupled circuits [8] are used. Indicators such as state-ofcharge (SOC), state-of-health, and internal temperature are design considerations for various charging strategies based on offline methods, such as frequency optimization [9], dynamic programming [10], genetic algorithm [11], as well as online methods such as fuzzy control [12] and model predictive control (MPC) [13]. The target is usually set to achieve the maximum charging efficiency [14] or the minimum charging loss during fast charging [10]. Since these indicators cannot be measured directly, state observers, such as those based on Kalman filters [15], $H$-infinity observers [11], and recursive least squares [16], are needed to estimate the internal states and indicators online. However, as the conventional ECMs are empirically developed based on external measurements, usually under normal operating conditions with low-to-medium current rates, it is difficult to accurately capture the battery characteristics and predict the battery operating limits during fast charging in which both the current and temperature ranges are much wider. Extrapolation of the degradation behaviors is prone to fail due to the lack of mechanistic description of aging phenomena such as the growth of the solid-electrolyte interface (SEI) film and lithium plating [2].

Physics-based models have thus recently been investigated for the design of fast charging algorithms. These models describe electrochemical phenomena, such as ion diffusion, intercalation kinetics, as well as heat generation/heat transfer, and they are capable of reproducing battery dynamics with high fidelity [17]. Based on physics-based models, optimization problems and open-loop optimal controls have been formulated to minimize charging duration [18]. These results can be regarded as a first step toward the design of closed-loop controllers that are able to compensate for model uncertainties and disturbances. A few charging algorithms equipped with closed-loop control algorithms based on physics-based models were recently proposed. For instance, a fast-charging strategy was developed utilizing an isothermal electrochemical model, health-related constraints, and nonlinear MPC (NMPC) [19]. By incorporating thermal dynamics into a multi-physics model described by partial-differential-algebraic equations (PDAEs), a reference governor and one-step NMPC were proposed to optimize charging profiles [20]. However, the applications are potentially limited by their intractable computations associated with nonlinear PDAE models and nonlinear online optimization [21]. To solve the problem of low computational efficiency, an NMPC was proposed based on a reduced-order model of a Li-ion battery [21], where the distributed effect along the electrodes of the reduced-order model was completely ignored. However, recent evidence has shown that lumping the behaviors in the electrode can lead to an underestimation of Li-ion battery degradation during fast charging [22].

In this connection, it will be shown that ignoring the nonuniform charge and mass distribution in the electrodes of the Li-ion batteries can cause significant modeling errors and make the designed control scheme less effective during fast charging. This is in particular true for today's high-energy type battery cells with wider electrodes and high-power type battery cells with increased rate capability. To address this problem, we first use spatial discretization techniques to reduce a PDAEbased multi-physics battery model into a differential-algebraic equation system, so that the spatially distributed degradationand safety-related dynamics can be accurately captured. By reformulating the reduced model next into a physics-based equivalent circuit network with only ordinary differential e- 
quations (ODEs), the model can be efficiently solved, while the internal dynamics can be better illustrated and explained for researchers with electrical engineering backgrounds. To our best knowledge, this is the first work using a spatially discretized physics-based equivalent circuit model for Li-ion battery charging control design [2].

In the scenario of high current-rate charging, the necessity to adequately discretize the nonlinear PDAE-based distributedparameter battery model inevitably leads to a high-order system. This creates numerical issues in stochastic state estimation: It is unaffordable for many nonlinear state estimation algorithms, such as the extended Kalman filter (EKF), the unscented Kalman filter (UKF), a particle filter (PF), etc., although all of these algorithms based on the discretized battery electrochemical model have been theoretically studied for distributed state estimation purposes, see [23]-[25]. To address such a computational difficulty, the ensemble Kalman filter (EnKF) was adopted in [26] and showed superior efficiency for the high-order systems. EnKF is a sequential Monte Carlo implementation of the Kalman filter by using low dimension random samples (i.e., an ensemble) to represent statistics of the model uncertainty. Nevertheless, in practice, the EnKF can suffer from reduced accuracy and even divergence when a relatively small set of samples are selected [27]. In order to overcome this issue, an improved ensemble-based algorithm, named the ensemble transfer Kalman filter (ETKF) [28], is introduced for the first time to effectively enhance the performance of distributed state estimation for the battery system, and this forms the first major contribution of the present work.

Again, due to the high-order nature of the system model, online optimization-based control methods, such as NMPC, are hardly implementable for battery fast charging. This is popularly known as the "curse of dimensionality". In order to overcome this obstacle, we design a simple fast-charging scheme that regulates the rate-limiting constraints using proportionalintegral (PI) feedback control while maximizing the charging speed. In this way, the objectives in terms of charging time, degradation, and battery safety can be properly balanced. The used battery model does not relay on the uniform distribution hypothesis of some electrode states that commonly used in the literature and accurately predicts internal distributed dynamics during fast charging. The proposed optimization-free charging forms the second major contribution.

\section{Physics-Based Battery Modeling}

The pseudo-two-dimensional model with thermal dynamics (P2D-T) is considered to be one of the most precise mathematical descriptions of Li-ion batteries, and it can accurately exhibit the internal health- and safety-related behavior under a wide operating range [17]. Since the P2D-T model is a nonlinear PDAE system, model order reduction must be carried out so that an ODE system with a finite number of state variables is used. We resort to the finite volume method (FVM) to retain the distributed characteristics of the cell. In contrast to many ad-hoc methods, such a concept is mathematically mature and applicable to any operating condition.

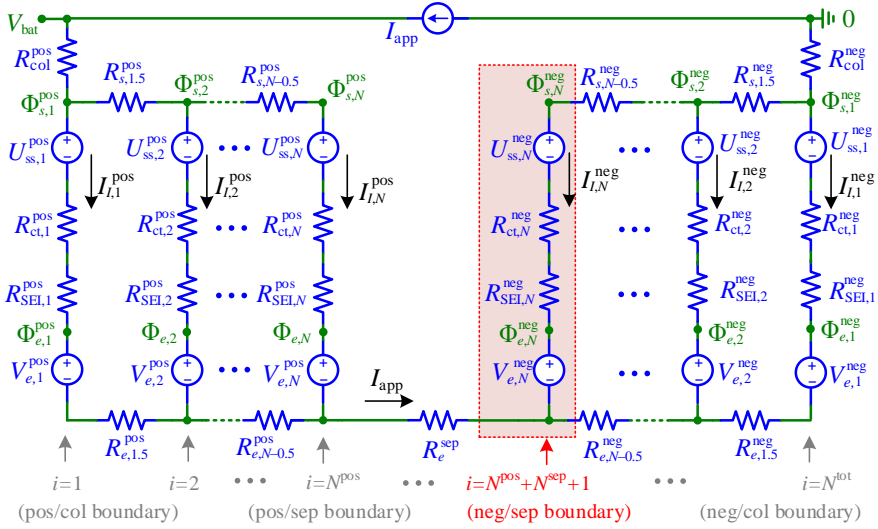

Fig. 1. Equivalent circuit describing the charge transport in the solid-phase, charge transport in the electrolyte, and the electrochemical reaction kinetics. In the $i$ th control volume, $V_{e, i}$ is controlled by $c_{e, i}$ in Fig. 2(a), and $U_{\mathrm{ss}, i}$ is controlled by $c_{\mathrm{ss}, i}$ in Fig. 2(b), respectively.

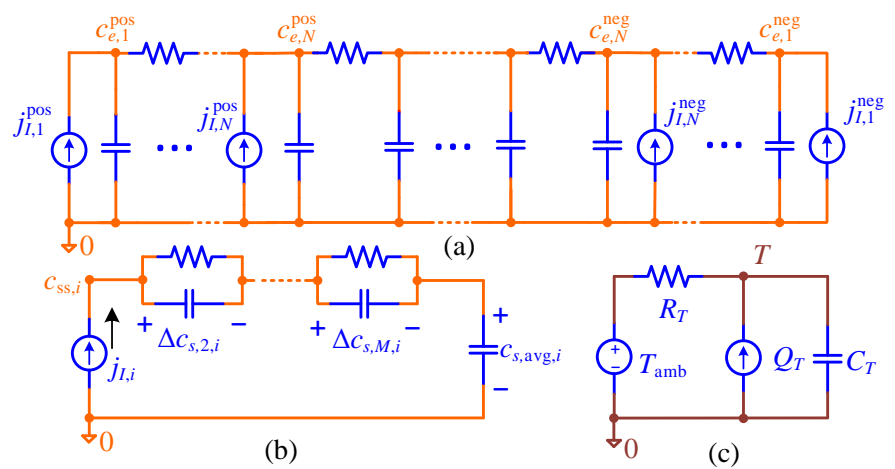

Fig. 2. Equivalent circuit describing (a) diffusion in the electrolyte, (b) diffusion in the solid phase in the $i$ th control volume, and (c) thermal dynamics. In the $i$ th control volume, $j_{I, i}$ is controlled by $I_{I, i}$ in Fig. 1.

Using the framework proposed in [17], the equations related to charge transport phenomena can be converted into an equivalent subcircuit as shown in Fig. 1. Here $V_{\text {bat }}$ and $I_{\text {app }}$ are the cell voltage across and charging current flowing through the two current collectors (col) respectively. The space across the horizontal cell thickness is divided into several control volumes, with $N^{\text {pos }}, N^{\text {sep }}$, and $N^{\text {neg }}$ being the numbers of the control volumes of the positive electrode (pos), the separator (sep), and the negative electrode (neg), respectively. Subscript $i \in\left\{1,2, \cdots, N^{\text {tot }}=N^{\text {pos }}+N^{\text {sep }}+N^{\text {neg }}\right\}$ is used to indicate the local quantity at the central node of the $i$ th control volume, while $i \pm 0.5$ represents the quantity at the edges of the control volume. For ease of notation, a local quantity $X_{i}$ in the positive electrode is denoted by $X_{i}^{\text {pos }}, \forall i \in \mathcal{S}^{\text {pos }}:=$ $\left\{1, \cdots, N^{\text {pos }}\right\}$. While in the negative electrode, $X_{i}$ is denoted by $X_{N^{\text {tot }}-i+1}^{\text {neg }}, \forall i \in \mathcal{S}^{\text {neg }}:=\left\{N^{\text {pos }}+N^{\text {sep }}+1, \cdots, N^{\text {tot }}\right\}$. In addition, considering that the widths of the two electrodes are usually comparable, it is assumed that $N^{\text {pos }}=N^{\text {neg }}=N$ in this work to simplify the analysis and notation. The physical meanings of all the symbols used in Fig. 1 and in the remaining part of the work are given in the Nomenclature.

Mass transport phenomena are modeled by the proposed subcircuits shown in Figs. 2(a) and (b). These two equivalent 
circuits approximate Fick's law of diffusion equations that govern the variations of electrolyte concentration $c_{e, i}$ and solid-phase surface concentration $c_{\mathrm{ss}, i}$, respectively. These concentration states are directly analogized as capacitor voltages, while molar fluxes $j_{I, i}$ (which are proportional to the branch currents $I_{I, i}$ in Fig. 1) are analogized as capacitor currents. This is in contrast to using nonlinear transformations as in [17], so that higher computation efficiency can be achieved. Furthermore, Fig. 2(a) is obtained using FVM, whereas in Fig. 2(b), Padé approximation is used for reduced computational burden [29]. In Fig. 2(b), $c_{s, \text { avg }, i}$ is the volume-averaged solid-phase concentration, and $\Delta c_{s, l, i}$ for $l \in\{2, \cdots, M\}$ are $M-1$ concentration difference terms. The SOC of the cell is associated with all $c_{s, \text { avg }, i}$ in the negative electrode, i.e.,

$$
\operatorname{SOC}(t)=\frac{\frac{1}{N^{\text {neg }}} \sum_{i \in \mathcal{S}^{\text {neg }}} c_{s, \text { avg }, i}(t)-c_{s, 0 \%}^{\text {neg }}}{c_{s, 100 \%}^{\text {neg }}-c_{s, 0 \%}^{\text {neg }}} .
$$

In Fig. 1, each vertical branch has two resistances, i.e., the SEI film resistance $R_{\mathrm{SEI}, i}$ and the charge-transfer resistance $R_{\mathrm{ct}, i}$, and they are closely related to battery degradation. SEI film growth and lithium plating (LiP) are the two major degradation mechanisms of Li-ion batteries during the charging process [30], which can lead to aging phenomena such as capacity fade and increase of internal resistance. As identified in the literature and by experiment, both of these two types of side reactions occur most severely at the negative electrode/separator (neg/sep) boundary as indicated in Fig. 1. At this boundary, the rates of the side reactions are determined by the side-reaction potentials (SRP), i.e., $\forall$ sr $\in\{\mathrm{SEI}, \mathrm{LiP}\}$,

$$
\eta_{\mathrm{sr}, N}^{\mathrm{neg}}(t)=U_{\mathrm{ss}, N}^{\mathrm{neg}}(t)+R_{\mathrm{ct}, N}^{\mathrm{neg}} I_{I, N}^{\mathrm{neg}}(t)-U_{\mathrm{sr}}^{\mathrm{ref}}
$$

where $U_{\mathrm{sr}}^{\mathrm{ref}}$ is the reference voltage for the side reactions. The charge-transfer resistance $R_{\mathrm{ct}, N}^{\text {neg }}$ is determined by $c_{\mathrm{ss}, N}^{\text {neg }}, c_{e, N}^{\text {neg }}$, as well as cell temperature $T$, and their relationship is [17]

$$
R_{\mathrm{ct}, N}^{\mathrm{neg}} \propto T(t) / \sqrt{c_{e, N}^{\mathrm{neg}}(t) c_{\mathrm{ss}, N}^{\mathrm{neg}}(t)\left(c_{s, \text { max }}^{\mathrm{neg}}-c_{\mathrm{ss}, N}^{\mathrm{neg}}(t)\right)}
$$

where $c_{s, \text { max }}^{\text {neg }}$ is the theoretical maximum concentration in the solid phase of the negative electrode.

To describe the temperature dynamics, the lumped thermal circuit shown in Fig. 2(c) is adopted. Here, $R_{T}$ is the thermal resistance, $C_{T}$ is the thermal capacitance, $T_{\mathrm{amb}}$ is the ambient temperature, and $Q_{T}$ is the heat source term. The thermal model is coupled with the electrochemical models: $Q_{T}$ is calculated using Fig. 1 as the sum of the irreversible heat generated by the resistances and the reversible heat generated by the capacitances. $T$ in Fig. 2(c), in turn, affects the parametric values of the elements in all other subcircuits. More details about the mathematical expressions and physical meanings of the symbols are provided in [17], [26].

We denote the circuit model in Fig. 1 and Fig. 2 the multiphysics circuit model (MPCM) in the latter sections of the paper. After being discretized in the time domain, the MPCM can be expressed in a discrete-time state-space form,

$$
\begin{aligned}
\mathbf{x}_{k} & =\mathcal{F}\left(\mathbf{x}_{k-1}, u_{k}, \Delta t\right)+\mathbf{w}_{k} \\
\mathbf{z}_{k} & =\mathcal{H}\left(\mathbf{x}_{k}, u_{k}\right)+\mathbf{v}_{k} \\
\mathbf{y}_{k} & =\mathcal{G}\left(\mathbf{x}_{k}, u_{k}\right)
\end{aligned}
$$

where $\mathcal{F}(\cdot), \mathcal{H}(\cdot)$, and $\mathcal{G}(\cdot)$ are nonlinear operators, $u=$ $I_{\text {app }}$ is the single control input, and $\mathbf{z}=\left[V_{\mathrm{bat}}, T_{\mathrm{amb}}\right]^{\top}$ is the measurement vector. The state vector $\mathbf{x}=$ $\left[\left(\mathbf{c}_{s, \text { avg }}^{\text {pos }}\right)^{\top},\left(\mathbf{c}_{s, \text { avg }}^{\text {neg }}\right)^{\top},\left(\mathbf{c}_{e}\right)^{\top},\left(\Delta \mathbf{c}_{s}\right)^{\top}, T\right]^{\top} \in \mathbb{R}^{n}$ consists of all the concentrations and the temperature (i.e., the voltages of all the equivalent capacitors shown in Fig. 2), and the system order is $n=M\left(N^{\text {pos }}+N^{\text {neg }}\right)+N^{\text {tot }}+1$. The uncertainties of the process and measurements are considered in (3) by hypothesized normally distributed error vectors $\mathbf{w} \sim \mathcal{N}(0, \mathbf{Q})$ and $\mathbf{v} \sim \mathcal{N}(0, \mathbf{R})$, respectively. Furthermore, the output vector $\mathbf{y}$ to be controlled includes variables that will be used as the constraints in the fast charging strategy, e.g., SRPs, SOC, temperature, and concentrations. These constraints will be estimated using the distributed battery state estimator proposed next.

\section{Distributed BAtTERy StATE Estimation}

As will be presented later in Section V-A, a sufficiently large number of control volumes are needed to accurately predict the constraints that affect battery charging control design. In this section, an estimator is therefore proposed to observe the distributed states of the Li-ion batteries based on the MPCM.

Due to the fact that the MPCM possesses a much higher order compared to the conventional ECMs, nonlinear estimators based on UKF, EKF, or PF algorithms can suffer problems of high computational overhead. This is because, in these algorithms, both an $n$-dimensional mean vector and an $n$-by- $n$ error covariance matrix of the state variables need to be evolved in real-time [27]. Instead, in ensemble-based algorithms such as EnKF, sampled state vectors are adopted to represent the state mean and the error covariance [28]. The number $m$ of the state vector samples, namely the ensemble size, can be selected much smaller than the system order $n$ so that the online implementation is computationally viable. However, as $m$ reduces, the EnKF algorithm tends to suffer the undersampling problem in which the error covariance will be underestimated [27]. This issue is caused by the introduction of the pseudo-random measurement perturbation in the update step. In this connection, an MPCM-based battery distributed state estimator is proposed in this section by incorporating the deterministic ETKF algorithm introduced in [28]. An ensemble initialization process will be proposed first for the battery state estimator, after which the governing equations of the estimator will be presented based on the ETKF algorithm described in [28].

\section{A. Ensemble Initialization}

A state ensemble is an $n$-by- $m$ matrix, and each column of it is an estimate of the state vector. The ETKF-based battery state estimator is first initialized by choosing an initial state ensemble $\hat{\mathbf{X}}_{0}^{+}:=\left[\hat{\mathbf{x}}_{0,1}^{+}, \hat{\mathbf{x}}_{0,2}^{+}, \ldots, \hat{\mathbf{x}}_{0, m}^{+}\right]$. Improper setting of $\hat{\mathbf{X}}_{0}^{+}$ can lead to slow initial convergence of the estimator [31]. If no other prior statistical information is available, $\hat{\mathbf{X}}_{0}^{+}$can be assumed to evenly cover an estimated range of SOC from $\mathrm{SOC}_{0}^{\min }$ to $\mathrm{SOC}_{0}^{\max }$. Accordingly, the element in the $q$ th row and the $p$ th column in $\hat{\mathbf{X}}_{0}^{+}$is set to 


$$
\hat{\mathbf{X}}_{0}^{+}(q, p)=\left\{\begin{array}{cc}
c_{s 0, p}^{\text {pos }}, & 1 \leq q \leq N^{\text {pos }} \\
c_{s 0, p}^{\text {neg }}, & N^{\text {pos }}+1 \leq q \leq N^{\text {pos }}+N^{\text {neg }} \\
c_{e 0}, & N^{\text {pos }}+N^{\text {neg }}+1 \leq q \leq N^{\text {pos }}+N^{\text {neg }}+N^{\text {tot }} \\
T_{0}, & q=n \\
0, & \text { otherwise }
\end{array}\right.
$$

where the initial solid-phase concentrations are obtained using

$$
\begin{aligned}
c_{s 0, p}^{\mathrm{pos}} & =\left(c_{s, 0 \%}^{\mathrm{pos}}-c_{s, 100 \%}^{\mathrm{pos}}\right) \mathrm{SOC}_{0, p}+c_{s, 100 \%}^{\mathrm{pos}} \\
c_{s 0, p}^{\mathrm{neg}} & =\left(c_{s, 100 \%}^{\text {neg }}-c_{s, 0 \%}^{\text {neg }}\right) \mathrm{SOC}_{0, p}+c_{s, 0 \%}^{\text {neg }} \\
\mathrm{SOC}_{0, p} & =\mathrm{SOC}_{0}^{\min }+\frac{p}{m}\left(\mathrm{SOC}_{0}^{\max }-\mathrm{SOC}_{0}^{\min }\right) .
\end{aligned}
$$

In (4a), the initial electrolyte concentration $c_{e 0}$ is a known battery parameter determined by the material characteristics of the electrolyte, while the initial temperature $T_{0}$ is measurable and normally close to the ambient temperature $T_{\text {amb }}$.

\section{B. Prediction Step}

The ETKF is similar to the EnKF in that they both share the same prediction step. Based on the previous posterior state ensemble $\hat{\mathbf{X}}_{k-1}^{+}:=\left[\hat{\mathbf{x}}_{k-1,1}^{+}, \hat{\mathbf{x}}_{k-1,2}^{+}, \ldots, \hat{\mathbf{x}}_{k-1, m}^{+}\right]$, the new prior state ensemble $\hat{\mathbf{X}}_{k}^{-}:=\left[\hat{\mathbf{x}}_{k, 1}^{-}, \hat{\mathbf{x}}_{k, 2}^{-}, \ldots, \hat{\mathbf{x}}_{k, m}^{-}\right]$is obtained according to the state equation (3a). Thus, $\forall p \in\{1,2, \cdots, m\}$,

$$
\hat{\mathbf{x}}_{k, p}^{-}=\mathcal{F}\left(\hat{\mathbf{x}}_{k-1, p}^{+}, u_{k}, \Delta t\right)+\mathbf{w}_{k, p} .
$$

The prior ensemble mean $\overline{\mathbf{x}}_{k}^{-}$and the prior ensemble perturbation $\tilde{\mathbf{X}}_{k}^{-}$are defined as

$$
\begin{aligned}
& \overline{\mathbf{x}}_{k}^{-}:=\frac{1}{m} \sum_{p=1}^{m} \hat{\mathbf{x}}_{k, p}^{-} \\
& \tilde{\mathbf{X}}_{k}^{-}:=\hat{\mathbf{X}}_{k}^{-}-\overbrace{\left[\overline{\mathbf{x}}_{k}^{-}, \overline{\mathbf{x}}_{k}^{-}, \cdots, \overline{\mathbf{x}}_{k}^{-}\right]}^{m} .
\end{aligned}
$$

Next, the measurement ensemble is defined as $\hat{\mathbf{Z}}_{k}:=$ $\left[\hat{\mathbf{z}}_{k, 1}, \hat{\mathbf{z}}_{k, 2}, \ldots, \hat{\mathbf{z}}_{k, m}\right]$, and each column of $\hat{\mathbf{Z}}_{k}$ is obtained according to (3b), i.e., $\forall p \in\{1,2, \cdots, m\}$,

$$
\hat{\mathbf{z}}_{k, p}=\mathcal{H}\left(\hat{\mathbf{x}}_{k, p}^{-}, u_{k}\right) \text {. }
$$

Similar to (5a) and (5b), the ensemble mean and the ensemble perturbation of the measurements are defined by

$$
\begin{aligned}
& \overline{\mathbf{z}}_{k}:=\frac{1}{m} \sum_{p=1}^{m} \hat{\mathbf{z}}_{k, p} \\
& \tilde{\mathbf{Z}}_{k}:=\hat{\mathbf{Z}}_{k}-\overbrace{\left[\overline{\mathbf{z}}_{k}, \overline{\mathbf{z}}_{k}, \cdots, \overline{\mathbf{z}}_{k}\right]}^{m} .
\end{aligned}
$$

With the ensemble perturbation matrices (5c) and (5f), the ensemble Kalman gain $\mathbf{K}_{e, k}$ can be calculated by

$$
\mathbf{K}_{e, k}=\frac{\tilde{\mathbf{X}}_{k}^{-} \tilde{\mathbf{Z}}_{k}^{\top}}{m-1}\left(\frac{\tilde{\mathbf{Z}}_{k} \tilde{\mathbf{Z}}_{k}^{\top}}{m-1}+\mathbf{R}_{k}\right)^{-1} .
$$

\section{Update Step of EnKF}

The posterior state ensemble $\hat{\mathbf{X}}_{k}^{+}$in the EnKF is updated by

$$
\hat{\mathbf{X}}_{k}^{+}=\hat{\mathbf{X}}_{k}^{-}+\mathbf{K}_{e, k}\left(\mathbf{Z}_{k}+\boldsymbol{\Gamma}_{k}-\hat{\mathbf{Z}}_{k}\right)
$$

where $\boldsymbol{\Gamma}_{k}$ is named the pseudo measurement perturbation, and each column of $\boldsymbol{\Gamma}_{k}$ is normally distributed according to $\mathcal{N}\left(0, \mathbf{R}_{k}\right)$. By adding $\boldsymbol{\Gamma}_{k}$, the posterior covariance matrix approaches its true value as $m$ increases [27].

\section{Update Step of ETKF}

Although the accuracy of the EnKF-based estimator is theoretically comparable to the conventional Kalman filter with a sufficiently large ensemble size, in the present application of battery state estimation, $m$ needs to be much smaller than $n$ to increase the computational efficiency. In the case of using a very small $m$, the introduction of $\boldsymbol{\Gamma}_{k}$ in the EnKF may lead to a large estimation error and even cause divergence problems due to undersampling, i.e., the ensemble generated with random perturbation is too small to statistically represent the mean and the covariance [28]. In the ETKF, instead, we consider to update the ensemble mean and the ensemble perturbation separately. The process is described below.

First, the posterior ensemble mean is obtained in a way similar to the conventional Kalman filter, without using the pseudo-random measurement perturbation, i.e.,

$$
\overline{\mathbf{x}}_{k}^{+}=\overline{\mathbf{x}}_{k}^{-}+\mathbf{K}_{e, k}\left(\mathbf{z}_{k}-\overline{\mathbf{z}}_{k}\right) .
$$

The posterior ensemble perturbation, though, is considered to be a linear transformation of the prior ensemble perturbation, i.e.,

$$
\tilde{\mathbf{X}}_{k}^{+}=\tilde{\mathbf{X}}_{k}^{-} \mathbf{T}_{k}
$$

where the transform matrix $\mathbf{T}_{k}$ is calculated by

$$
\mathbf{T}_{k}=\mathbf{U}_{k}\left(\mathbf{I}_{m}+\boldsymbol{\Lambda}_{k}\right)^{-\frac{1}{2}} \mathbf{U}_{k}^{\top} .
$$

Here, $\mathbf{I}_{m}$ is the identity matrix. $\boldsymbol{\Lambda}_{k}$ and $\mathbf{U}_{k}$ are obtained by performing the following eigendecomposition:

$$
\left(\tilde{\mathbf{Z}}_{k}^{\top} \mathbf{R}_{k}^{-1} \tilde{\mathbf{Z}}_{k}\right) /(m-1)=\mathbf{U}_{k} \boldsymbol{\Lambda}_{k} \mathbf{U}_{k}^{\top} .
$$

The posterior state ensemble is simply obtained by

$$
\hat{\mathbf{X}}_{k}^{+}=\overbrace{\left[\overline{\mathbf{x}}_{k}^{+}, \overline{\mathbf{x}}_{k}^{+}, \cdots, \overline{\mathbf{x}}_{k}^{+}\right]}^{m}+\tilde{\mathbf{X}}_{k}^{+} .
$$

Finally, with updated ensemble mean and the input, the unmeasurable outputs $\mathbf{y}_{k}$ can be estimated with (3c), i.e.,

$$
\hat{\mathbf{y}}_{k}=\mathcal{G}\left(\overline{\mathbf{x}}_{k}^{+}, u_{k}\right) .
$$

The ETKF algorithm presented above is much more computationally efficient to implement than the nonlinear benchmark algorithms such as EKF and UKF, especially when $m \ll n$. This is because only an $n$-by- $m$ ensemble matrix $\hat{\mathbf{X}}_{k}^{+}$need to be maintained and evolved. While for EKF and UKF, both the mean $\overline{\mathbf{x}}_{k}^{+}$of the state estimate and the $n$-by- $n$ covariance matrix $\hat{\mathbf{P}}_{k}^{+}$needs to be dealt with. Also, ETKF is advantageous over EKF as there is no need to perform sequential linearization, in which calculating a set of $n$-by- $n$ 
Jacobian matrices online can be extremely heavy for the highdimensional nonlinear model of the battery. Furthermore, the update step of the ETKF differs from the EnKF as there is no need to generate the pseudo-random measurement perturbation $\boldsymbol{\Gamma}_{k}$ in (6). This increases the accuracy and improves the numerical stability of the algorithm especially when a small ensemble is preferable. Although additional steps (7b)-(7d) including eigendecomposition are introduced in ETKF, the dimensions of relevant matrices, including $\mathbf{T}_{k}, \mathbf{I}_{m}, \boldsymbol{\Lambda}_{k}$, and $\mathbf{U}_{k}$, are only $m$-by- $m$. The effectiveness of the ETKF for battery electrochemical state estimation will be verified by a comparative study in Section V-A.

\section{FAST-CHARGING STRATEGY}

\section{A. Constraints for Li-Ion Battery Charging}

Many constraints have been considered to limit the charging rate when designing a fast charging strategy for Li-ion batteries in the literature. First, current $I_{\text {app }}$, voltage $V_{\text {bat }}$, and terminal power $P_{\text {bat }}=V_{\text {bat }} I_{\text {app }}$ of the battery are limited by the rating of the charging devices, i.e.,

$$
\begin{aligned}
& I_{\text {app }, k} \leq I_{\max } \\
& V_{\text {bat }, k} \leq V_{\max } \\
& P_{\text {bat }, k} \leq P_{\max }
\end{aligned}
$$

Second, due to the limited diffusivities, the concentrations in both the solid phase and the electrolyte can show significant nonuniformity. As will be shown in Fig. 7 and Fig. 8, in the negative electrode, the solid-phase and the electrolyte concentrations tend to saturate first at the neg/sep boundary and at the negative electrode/current collector (neg/col) boundary, respectively, compared to other locations during the charging process. To avoid local overcharge and lithium depletion, it is required to ensure

$$
\begin{aligned}
c_{\mathrm{ss}, N, k}^{\text {neg }} & <c_{s, \text { max }}^{\text {neg }} \\
-c_{e, 1, k}^{\text {neg }} & <0 .
\end{aligned}
$$

Third, side reactions will accelerate dramatically under high current rates for aged batteries due to the increased magnitude of the SRPs. To limit the side reactions, the SRP at the neg/sep boundary should be regulated above a certain minimum threshold $\eta_{\mathrm{sr}, \min }$, i.e., $\forall$ sr $\in\{\mathrm{SEI}, \mathrm{LiP}\}$,

$$
-\eta_{\mathrm{sr}, N, k}^{\mathrm{neg}} \leq-\eta_{\mathrm{sr}, \min }
$$

Finally, a large amount of heat will be generated during fast charging, and high temperature rise due to heat accumulation can cause safety issues such as thermal runaway. Assuming $T_{\max }$ is the maximum temperature limit for safe operation, thus

$$
T_{k} \leq T_{\max }
$$

must hold.

\section{B. Nonlinear Model Predictive Control}

Denote $\mathrm{SOC}_{0}$ the initial SOC and $\mathrm{SOC}_{f}$ the fully charged SOC. The general control objective of fast charging is to achieve the minimum charging time $t_{f}$ from $\mathrm{SOC}_{0}$ to $\mathrm{SOC}_{f}$, while the operating constraints as alluded to in Section IV-A are not violated. For real-time charging control, NMPC is an attractive candidate approach. NMPC repetitively solves the following nonlinear optimization problem (denoted as Problem 1) over a reduced prediction horizon $H<t_{f} / \Delta t$, and then only apply the first control action, i.e., at the time step $k$, determine the control sequence $\mathbf{I}_{\mathrm{app}, k}=$ $\left[I_{\text {app }, k}, I_{\text {app }, k+1}, \cdots, I_{\text {app }, k+H}\right]$, such that

Problem 1 (General Optimal Fast Charging):

$$
\min _{\mathbf{I}_{\text {app }, k}} \sum_{i=k}^{k+H}\left(\mathrm{SOC}_{i}-\mathrm{SOC}_{f}\right)^{2}
$$

subject to: $\quad$ system model (3) and state vector $\mathbf{x}_{k}$

$$
\text { constraints }(8)
$$

The entire charging process stops when $\mathrm{SOC}_{k} \geq \mathrm{SOC}_{f}$. As described in [20], NMPC is superior to global optimization approaches in terms of implementability. Furthermore, the uncertainty in the measurements and estimated states can be taken into account by incorporating a battery state estimator. However, existing NMPC-based fast-charging strategies rely on simplified surrogate low-order models. As will be shown in the next section, to accurately reflect the distributed behaviors of the battery during fast charging and predict the operating limits, a much higher system order is required. While the existing model-free approaches, such as CCCV and MCC, are not capable of fully addressing the constraints in (8), the NMPC-based algorithms will not be implementable due to the "curse of dimensionality" if the high-fidelity battery model (3) presented in Section II is to be adopted.

\section{General Physical Constraint-Triggered Feedback Control}

As will be shown in Section V-C, the NMPC results exhibit that although the optimized current profile is limited by various constraints at different charging stages, only one constraint comes into effect at a time. In this connection, a simple-toimplement fast charging strategy is proposed based on this fact. With a nonlinear function $G_{\lambda}(\cdot)$, a general output-error injection-based control law is proposed to calculate $I_{\mathrm{app}, k+1}$,

$$
I_{\text {app }, k+1}=G_{\lambda}\left(e_{\lambda, k}\right) \triangleq G_{\lambda}\left(y_{\lambda, k}-y_{\max , \lambda}\right)
$$

where $\lambda \in\{1,2, \cdots\}$ represents the index of the active constraint or the charging mode, $y_{\lambda, k}$ is an output variable on the LHS of (8) as well as a member of $\mathbf{y}_{k}$ in (3c), while $y_{\max , \lambda}$ is the corresponding maximum limit on the RHS of (8). It is worth mentioning that the formulation in (10) is essentially a governor of constraints or references if timevarying constraints have been predefined and used in (8). The control law is sufficiently general and can be embodied by a number of existing output-feedback controls, such as proportional-integral-differential and sliding mode controls.

Based on the above general physical constraint-triggered feedback control law, as an example, we will only consider a 
subset of the presented constraints in Section IV-A during the design of the charging strategy due to the following reasons.

1) Since most modern power electronic converters have a wide range of current and voltage variation capability, only the power rating $P_{\max }$ in (8c) is considered to be the external limiting factor.

2) Strictly speaking, (8d) and (8e) are descriptions of real battery characteristics governed by physical principles, rather than conditions to be controlled. These properties are reflected in the P2D-T model and preserved by the MPCM, as explained as follows. Consider the condition $c_{\mathrm{ss}, N}^{\text {neg }} \rightarrow c_{s, \text { max }}^{\text {neg }}$ or $c_{e, N}^{\text {neg }} \rightarrow 0$. According to (2b), the local charge-transfer resistance $R_{\mathrm{ct}, N}^{\text {neg }} \rightarrow \infty$, the corresponding vertical branch as shown in Fig. 1 is close to the open-circuit condition, and this leads to $I_{I, N}^{\text {neg }} \rightarrow 0$ and $j_{I, N}^{\text {neg }} \rightarrow 0$. Hence, according to Figs. 2(a) and (b), the further increase in $c_{\mathrm{ss}, N}^{\mathrm{neg}}$ or $c_{e, N}^{\mathrm{neg}}$ is approaching zero. Hence, (8d) and (8e) are automatically satisfied with the MPCM. In contrast, when designing the charging strategy using a battery model with lumped electrode and approximated electrolyte diffusion dynamics, such as [21], since the simulated concentrations can violate the constraints, (8d) and (8e) must be applied, and this can lead to a conservative design outcome.

3) Existing research works have identified that lithium plating occurs during fast charging with high current rates, which can result in significant lithium deposition at the neg/sep boundary. The influence on degradation due to lithium plating is dominant and much more significant than that due to the SEI film growth [22]. Hence, only the lithium plating is to be considered, i.e., in (8f), $\mathrm{sr}=\mathrm{LiP}$.

A switch-over rule can be designed to actively detect the violation of one of the constraints (8c), (8f), and (8g). If a violation occurs, the corresponding control (10) is activated, and the control action applies until another violation occurs.

\section{Physical Constraint-Triggered PI Control}

In the present work, (10) is implemented using a PI feedback controller with scheduled gains and a reset mechanism as depicted in Fig. 3. A strategy is first designed to actively switch among the three operating modes, i.e.,

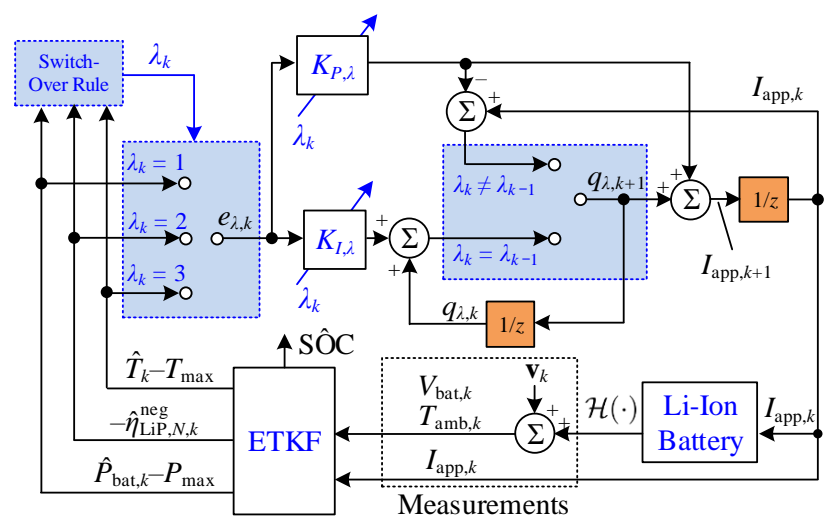

Fig. 3. Block diagram of the proposed physical constraint-triggered PI control for Li-ion battery fast charging.
Constant-power charging mode $(\lambda=1)$ : If the estimated power is equal to or higher than $P_{\max }$, the power PI control is active to prevent over-loading. Here, we consider that the true battery power is contaminated by measurement noises.

Zero-lithium-plating charging mode $(\lambda=2)$ : Maintaining LiP nonnegative can effectively restrain the occurrence of lithium plating [22]. If the estimated LiP potential at the neg/sep boundary reaches zero $\left(\eta_{\mathrm{LiP}, \min }=0\right)$, the LiP PI control is activated to restrain battery aging.

Constant-temperature charging mode $(\lambda=3)$ : If the cell temperature has reached the predefined upper limit $T_{\max }$, the temperature PI control is activated to avoid thermal runaway.

The PI control with the reset during the switch-over is expressed by

$$
\begin{aligned}
I_{\text {app }, k+1} & =K_{P, \lambda} \cdot e_{\lambda, k}+q_{\lambda, k+1} \\
q_{\lambda, k+1} & =\left\{\begin{array}{cc}
K_{I, \lambda} \cdot e_{\lambda, k}+q_{\lambda, k}, & \lambda_{k}=\lambda_{k-1} \\
I_{\text {app }, k}-K_{P, \lambda} \cdot e_{\lambda, k}, & \lambda_{k} \neq \lambda_{k-1}
\end{array}\right.
\end{aligned}
$$

where $K_{P, \lambda}$ and $K_{I, \lambda}$ are the proportional and integral control gains, respectively, and $q_{\lambda}$ is the output of the integrator. The integral state $q_{\lambda}$ is reset when the operating mode changes $\left(\lambda_{k} \neq \lambda_{k-1}\right)$ to ensure that $I_{\text {app }, k+1}=I_{\text {app }, k}$ and to achieve smooth switch-over processes. The battery SOC keeps increasing in all the three modes. If the estimated SOC has reached the predefined $\mathrm{SOC}_{f}$, the charging process terminates. This physical constraint-triggered PI (PCT-PI) control with particular switching rules is summarized as Algorithm 1.

The above three charging modes specifically consider the charging speed, aging rate, and safety level. This framework of the PCT-PI control is readily extendable to the fast charging of a Li-ion battery with different battery parameters and/or with other operating constraints, such as mechanical stress due to large concentration gradients [2]. The specific priority on the activation of different constraints and the corresponding switch-over rules are determined by the observation of the NMPC solution. In general, it is expected to charge with the maximum power so that the charging time is minimum. However, as will be shown in the latter section, the maximum power can only be applied at the initial stage of the charging process, before the activation of the lithium plating and the temperature constraint.

In this work, due to the high dimensionality and nonlinearity of the model, the PI control gains are obtained using trial-anderror methods. More rigorous tuning methods of the control parameters according to online estimated battery parameters shall be our future research focus.

Furthermore, the proposed method can also readily be extended to the conditions in which the effects of other degradation mechanisms are considered non-trivial. For example, side reactions on the positive electrode, such as decomposition of electrolyte and salt, can exponentially increase with the increasing voltage. The degradation rate and onset voltage depend on the stability of the electrolyte and passivation layer on the positive electrode. Once a suitable mathematical description has been established to predict the aging behavior, it can be incorporated into the presented battery model for the design of a revised constraint-triggered PI control strategy. 


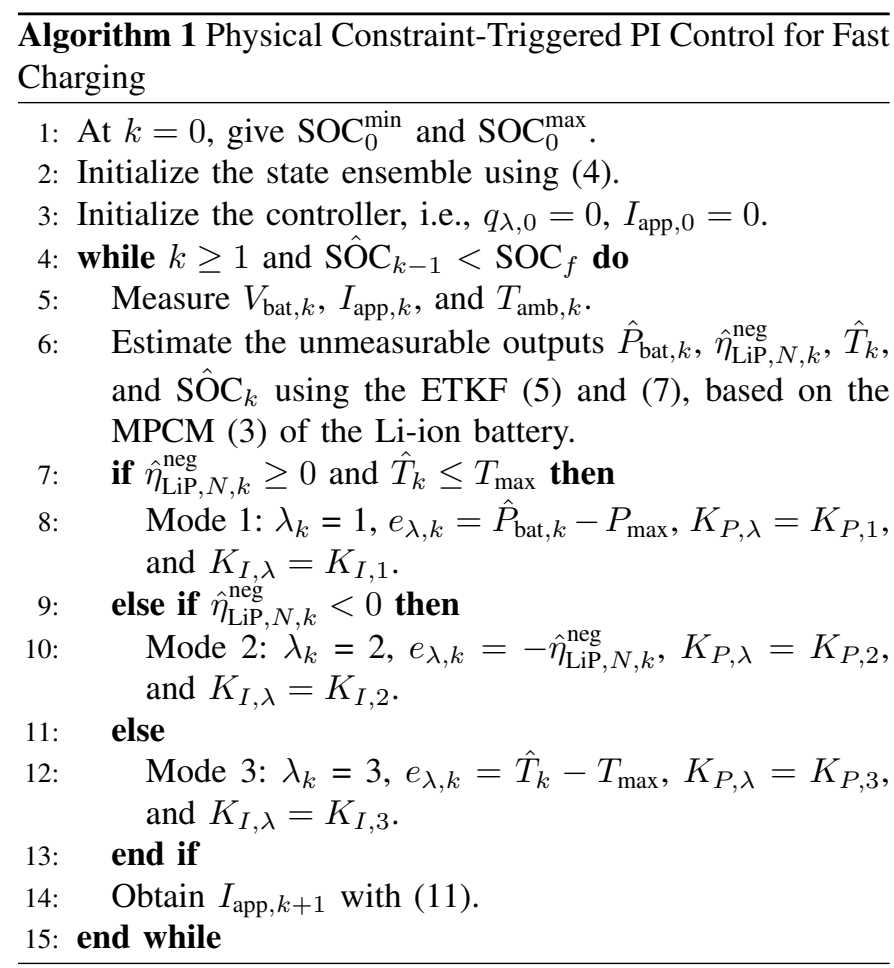

\section{Results AND Discussion}

\section{A. Model Validation and Comparison}

Experiments were conducted on a 3.5-Ah high-energy type lithium nickel manganese cobalt oxide (NMC) battery cell to validate the benchmark P2D-T model. A schematic of the experimental platform is provided in Fig. 4, including a BTS4000-5V6A battery tester with the maximum current of 6 A for power supply and current/voltage measurements, a workstation for user-machine interface and data acquisition, and a temperature aux unit with thermal resistor sensors. The cell was charged and discharged with various constant currents $(0.17 \mathrm{~A}, 1.7 \mathrm{~A}, 5.1 \mathrm{~A}$, and $6 \mathrm{~A})$ between the cut-over voltage $4.2 \mathrm{~V}$ and the cut-off voltage $2.5 \mathrm{~V}$, and the measured cell voltage and temperature are compared in Fig. 5 with the simulation results obtained using the P2D-T model. Here, the PDAE-based P2D-T model was implemented in COMSOL Multiphysics 5.3a on a PC with a processor @ $2.6 \mathrm{GHz}$ and 8GB RAM. Clearly, the P2D-T model reproduces the battery dynamics with high fidelity under a wide operating range. More validation results of the P2D-T model using high-power type cells can be found in various literature, such as [30]. Repetitive validation on cells with different types of chemistry is not represented.

Since it is impossible to measure the internal variables of the battery in real-time based on the prevailing sensor technology, we follow the common practice in the literature by comparing the MPCM with the validated benchmark model [21], [30]. In the process of verification of the internal behaviors during fast charging, all the battery parameters are taken from [32], corresponding to a 3-Ah high-power type lithium cobalt oxide cell. The MPCM was implemented in MATLAB R2016a environment with the same hardware configuration as

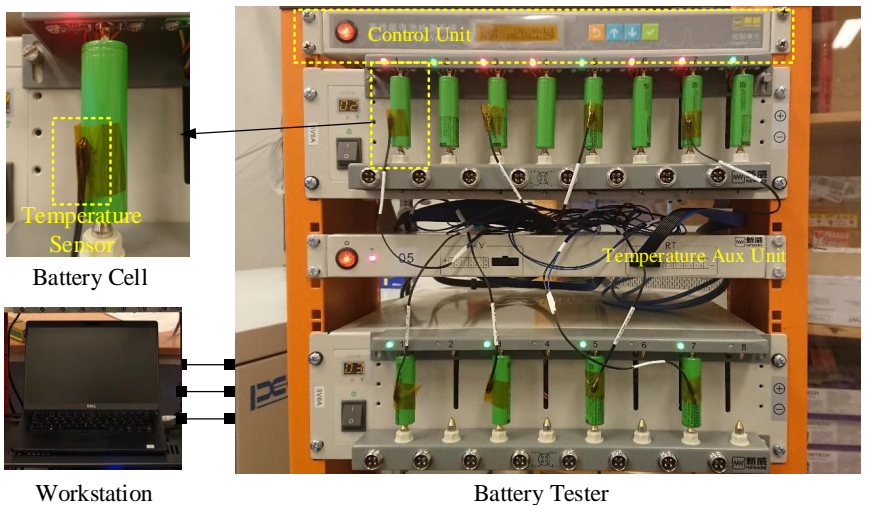

Fig. 4. Experimental platform for battery tests.
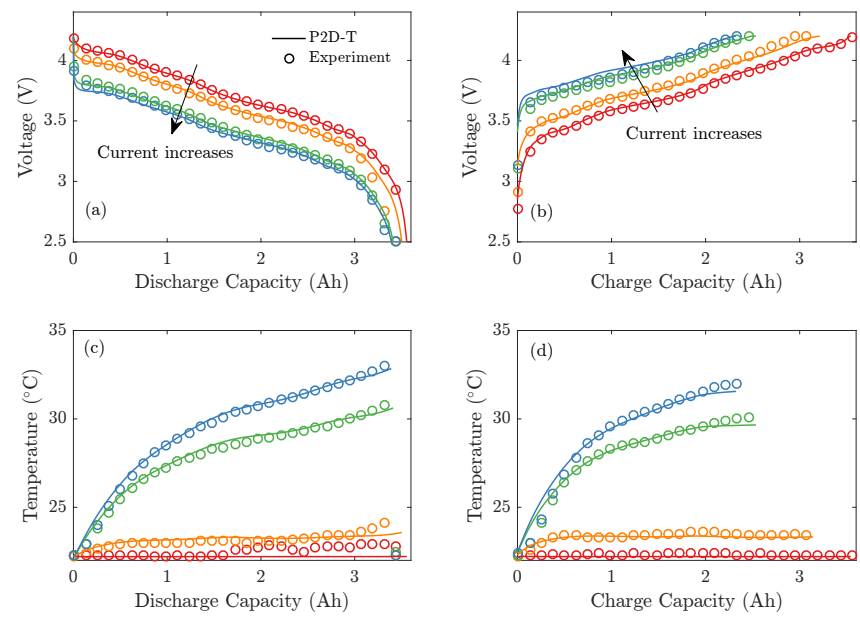

Fig. 5. Comparison of P2D-T model with experimentally measured terminal voltage and cell temperatures when the ambient temperature is $22^{\circ} \mathrm{C}$. (a) Voltage during discharge. (b) Voltage during charge. (c) Temperature during charge. (d) Temperature during discharge.

described in the previous paragraph. This configuration is also used for the validation of the proposed state estimation and control strategies in the next two subsections. The conventional CCCV and MCC protocols were applied with $4.2 \mathrm{~V}$ maximum terminal voltage. The simulated battery voltage, electrolyte concentrations, solid-phase surface concentrations, and LiP potentials are shown as functions of SOC or spatial position in Fig. 6 to Fig. 8, and the accuracy of the MPCM is summarized in Table I.

Unsurprisingly, it can be generally seen that, as the current rate increases, the model accuracy decreases with the same number of the control volumes. Fig. 6 shows that a small number of control volumes can lead to a significantly overestimated terminal voltage, especially under high current conditions ( $\geq 5 \mathrm{C}$ ). Fig. 7(a) shows that such a low-order model can cause underestimation of the LiP potential at the neg/sep boundary, and the error is mainly the direct result of the underestimated solid-phase and electrolyte concentrations at the same location, as presented in Figs. 7(b) and (c), respectively. Fig. 7(a) also indicates that $\mathrm{CCCV}$ and MCC cannot avoid lithium plating, since there are periods with $\eta_{\mathrm{LiP}, N}^{\text {neg }}<0$. This will hurt battery health and lead to premature battery degradation. 


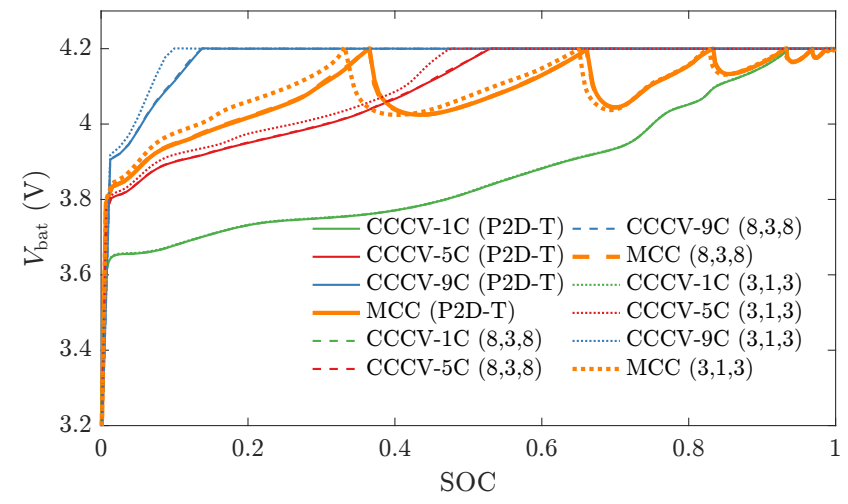

Fig. 6. Comparison of the simulated voltage with different charging protocols. Numbers within parentheses refer to control volumes $\left(N^{\text {pos }}, N^{\text {sep }}, N^{\text {neg }}\right)$ of the MPCM.

However, the accuracy of the model can always be increased by using a higher number of control volumes, and this is an advantage over most ad-hoc model reduction techniques whose validity depends on various assumptions. It can also be seen from Fig. 8 that under the extreme high current rate (9C) conditions, the gradient of the concentration and the distributed behaviors in the electrodes cannot be ignored, and that the spatial distribution is less uniform especially at the end of the charging process. Therefore, treating the profiles as uniform or approximating the profiles by a simple function can introduce significant errors for predicting the dynamics at the boundary. In addition, Fig. 8 shows the solid-phase concentrations at the pos/col boundary and the neg/sep boundary tend to approach the corresponding maximum limits during the fast charging process. Accordingly, accurately predicting the behaviors at these locations is important for the design of charging control strategies.

In addition, Fig. 7(b) and Fig. 8(a) show that the solidphase surface concentration is always below its theoretical maximum value $c_{s, \text { max }}^{\text {neg }}$. Similarly, Fig. 7(d) and Fig. 8(b) show that the simulated electrolyte concentration will never reach zero. These observations are in alignment with the mathematical analysis for inequality constraints (8d) and (8e) in Section IV-C.

TABLE I

PERFormanCE COMPARISON OF THE MPCM

\begin{tabular}{ccccc}
\hline \hline No. of & \multicolumn{4}{c}{ Terminal Voltage RMSE (\%) } \\
\cline { 2 - 5 } Control Volumes & CCCV & CCCV & CCCV & MCC \\
$\left(N^{\text {pos }}, N^{\text {sep }}, N^{\text {neg }}\right)$ & $(1 C)$ & $(5 C)$ & $(9 C)$ & \\
\hline$(15,5,15)$ & $<0.01$ & $<0.01$ & 0.012 & $<0.01$ \\
$(8,3,8)$ & 0.021 & 0.025 & 0.03 & 0.024 \\
$(5,2,5)$ & 0.085 & 0.10 & 0.25 & 0.09 \\
$(3,1,3)$ & 0.12 & 0.39 & 0.88 & 0.32 \\
\hline \hline
\end{tabular}

\section{B. Comparative Studies of Distributed State Estimators}

In order to demonstrate the efficacy of the ETKF-based distributed state estimator proposed in Section III, a modified federal urban driving schedule (FUDS) dynamic profile was used for its comparison with EnKF- and UKF-based algorithms. The original FUDS current profile [33] is repeated
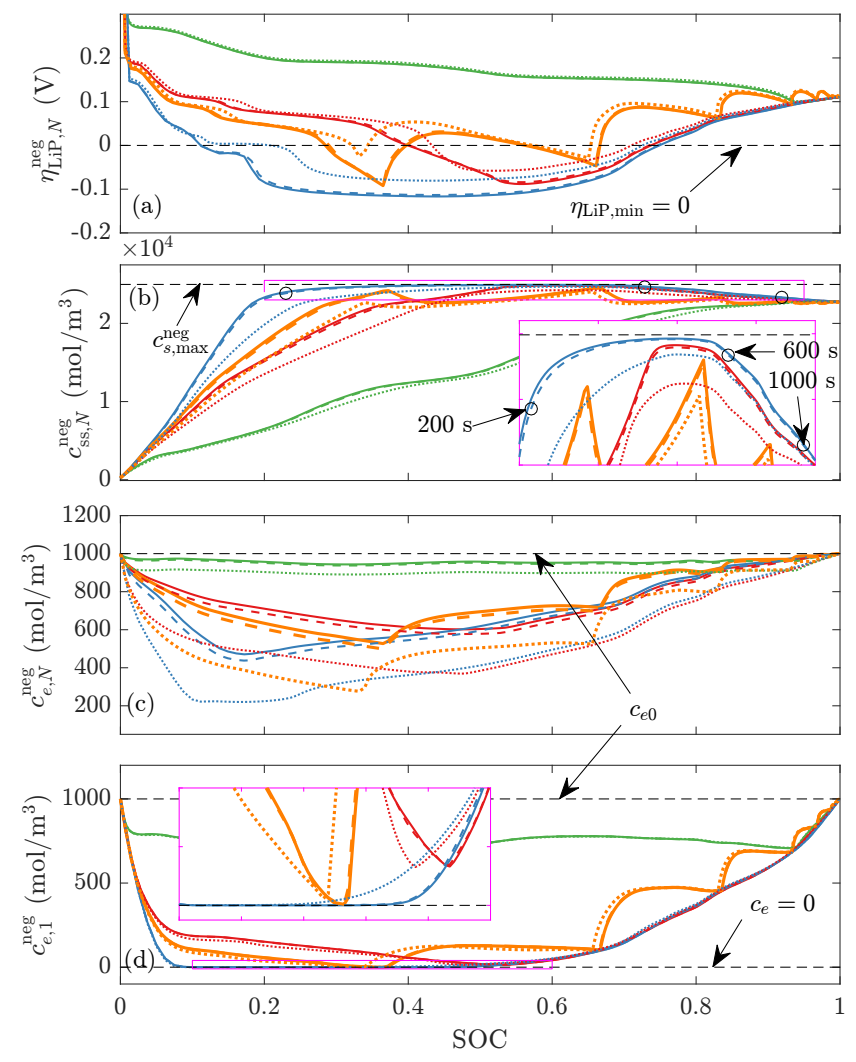

Fig. 7. Comparison of simulation results under different charging strategies: (a) LiP potential at the neg/sep boundary, (b) solid-phase surface concentration at the neg/sep boundary, (c) electrolyte concentration at the neg/sep boundary, and (d) electrolyte concentration at the neg/col boundary. Legend is the same as in Fig. 6.
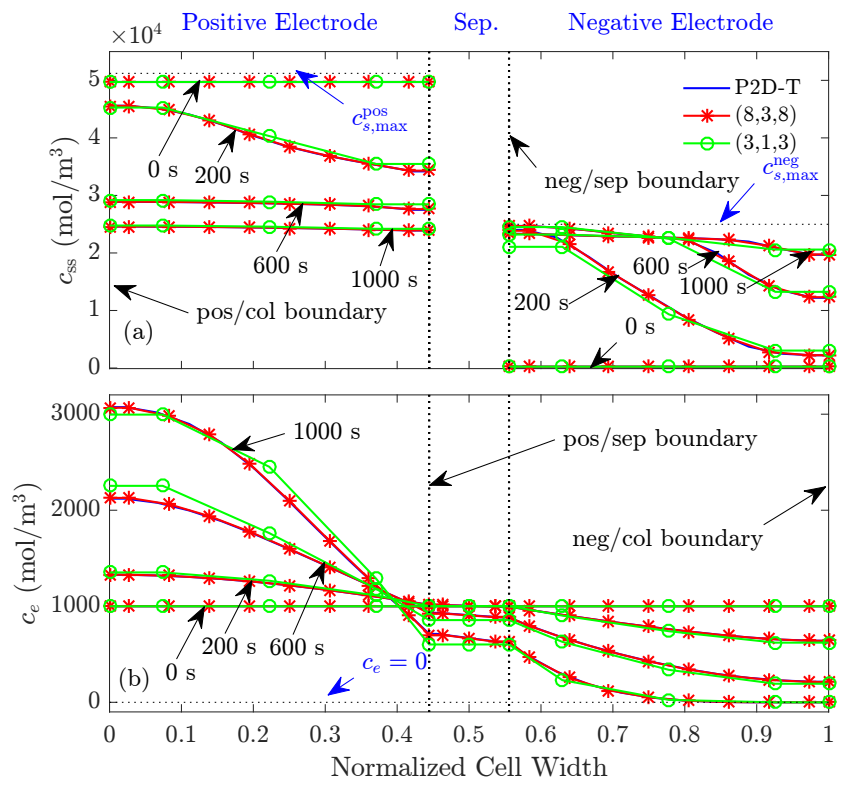

Fig. 8. Comparison of models under CCCV-9C charging: Spatial distribution of (a) the surface concentration in the solid phase, and (b) the electrolyte concentration.

twice and the current amplitude is increased with a maximum rate of $8 \mathrm{C}$. The reference data are again generated by the P2D$\mathrm{T}$ model. In the test, the numbers of the control volumes 
are $\left(N^{\text {pos }}, N^{\text {sep }}, N^{\text {neg }}\right)=(5,2,5)$, and the ensemble size is $m=3$. The standard deviations of the voltage and the temperature sensors are assumed to be $\pm 10 \mathrm{mV}$ and $\pm 1 \mathrm{~K}$, respectively, and thus the measurement noise covariance is $\mathbf{R}=\operatorname{diag}\left(\left[0.01^{2}, 1^{2}\right]\right)$. The initial SOC is set to 1.0 for all cases. To test the robustness and convergence speed of the employed estimation algorithms, a $50 \%$ initialization error in the SOC is considered. For the EnKF- and ETKF-based estimators, the state ensemble is initialized according to (4) with $\mathrm{SOC}_{0}^{\min }=0$ and $\mathrm{SOC}_{0}^{\max }=1$, so that the full SOC range is covered. Furthermore, the constraints on mass conservation in both the electrode and the electrolyte domains are included in the UKF algorithm to alleviate the slow divergence problem caused by the weak observability of the P2D model [25]. The sample time for the state estimators is chosen to be $\Delta t=0.5 \mathrm{~s}$.

The results of the estimated terminal voltage, SOC, and the solid-phase concentrations in the negative electrode are shown in Fig. 9, and performance metrics are compared in Table II. It can be seen that both the solutions of the EnKF and the ETKF converge rapidly towards their true values thanks to the proposed initialization steps described in Section III-A. For the UKF, though, Figs. 9(c) and (d) show that it takes $551 \mathrm{~s}$ to reach $1 \%$ SOC estimation error, which is much slower than the EnKF and the ETKF, even though the UKF has been enhanced with the consideration of mass conservation. The maximum absolute error (MAE) for SOC after convergence is $1.5 \%$ for the EnKF and its root mean square error (RMSE) is $0.93 \%$, whereas for the ETKF, the MAE and RMSE are only $0.34 \%$ and $0.13 \%$, respectively. The estimation errors of the surface concentrations in the negative electrode using the ETKF are also significantly smaller than that using EnKF, which can be observed in Fig. 9(f). Furthermore, the ETKF and the EnKF are much more computationally efficient than the UKF, as can be seen from Table II. The ETKF is slightly slower than the EnKF because of the additional steps needed to perform the eigendecomposition $(7 \mathrm{~d})$.

TABLE II

PERformance Comparison of Nonlinear State Estimators

\begin{tabular}{cccc}
\hline \hline & UKF & EnKF & ETKF \\
\hline Convergence Time* (s) & 551 & 2 & 2 \\
RMSE of SOC (\%) & 0.41 & 0.93 & 0.13 \\
MAE of SOC (\%) & 1.0 & 1.5 & 0.34 \\
CPU Time (s) & 256.4 & 22.6 & 25.1 \\
\hline \hline
\end{tabular}

\section{Comparative Studies of Fast Charging Strategies}

In this subsection, the fast charging strategy based on the PCT-PI control (Alg. 1) proposed in Section IV-D is compared with CCCV, MCC, and NMPC. The control gains for the proposed strategy are chosen by trial-and-error to be $K_{P, 1}=$ $0.01, K_{I, 1}=0.1, K_{P, 2}=10, K_{I, 2}=100, K_{P, 3}=30$, and $K_{I, 3}=0.3$. Various CCCV and MCC schemes were tested, all with a maximum voltage of $4.2 \mathrm{~V}$. The results of two CCCV schemes and an MCC scheme are presented and compared. The current rates in the CC stage of the CCCV schemes are $6 \mathrm{C}$ and $9 \mathrm{C}$, respectively, and the current sequence for the
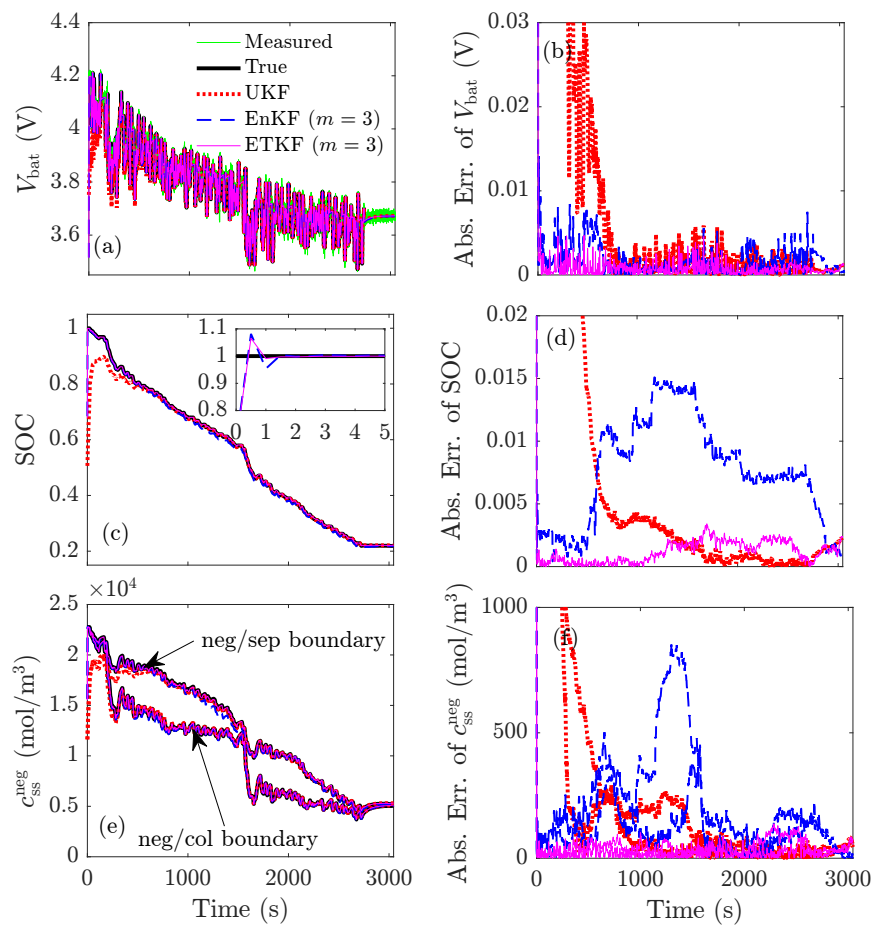

Fig. 9. Comparison of different nonlinear state estimators under a modified FUDS current profile with a maximum current rate of 8C: (a) Battery voltage, (b) voltage estimation error, (c) SOC, (d) SOC estimation error, (e) surface concentrations in the solid phase at the neg/sep boundary and at the neg/col boundary, and (f) solid-phase concentration estimation error.

MCC scheme is [7C, $4 \mathrm{C}, 2 \mathrm{C}, 1 \mathrm{C}, 0.5 \mathrm{C}, 0.2 \mathrm{C}, 0.1 \mathrm{C}]$. Other schemes only produce comparable performances in terms of charging time if the constraints are not significantly violated. For the NMPC, the prediction horizon is selected to be $5 \mathrm{~s}$ due to the heavy computational burden, and Problem 1 is solved using the fmincon function in MATLAB. The optimality can be improved by increasing the prediction horizon, and a similar design procedure can be carried out based on the proposed framework. As benchmark, the states in the NMPC are assumed perfectly measured without using an estimator, and the sample time is $1 \mathrm{~s}$ for all strategies. In order to obtain the unmeasurable internal states for online validation, in this case, the battery dynamics are simulated using the validated high-fidelity MPCM, which is also implemented in the MATLAB environment described earlier in Section V-A. Starting from time $t=0 \mathrm{~s}$, the simulation results are shown in Fig. 10, and the performance is summarized in Table III. For the proposed strategy, as can be seen from Fig. 10(d), from 0 to $38 \mathrm{~s}$ the battery was first charged with the maximum power $P_{\max }=120 \mathrm{~W}$ (Mode 1). From Fig. 10(e), it can be observed that the estimated LiP potential drops rapidly, and at the end of $38 \mathrm{~s}$, the LiP potential reaches zero. Hence, the charging switches to Mode 2 so that the degradation due to lithium plating is avoided. This mode lasts about $200 \mathrm{~s}$ until the cell temperature reaches the pre-set upper limit $T_{\max }=43^{\circ} \mathrm{C}$ at $245 \mathrm{~s}$, see Fig. $10(\mathrm{f})$. The temperature PI control is then activated (Mode 3) to limit the temperature rise by decreasing the charging current. In this mode, it can be seen 

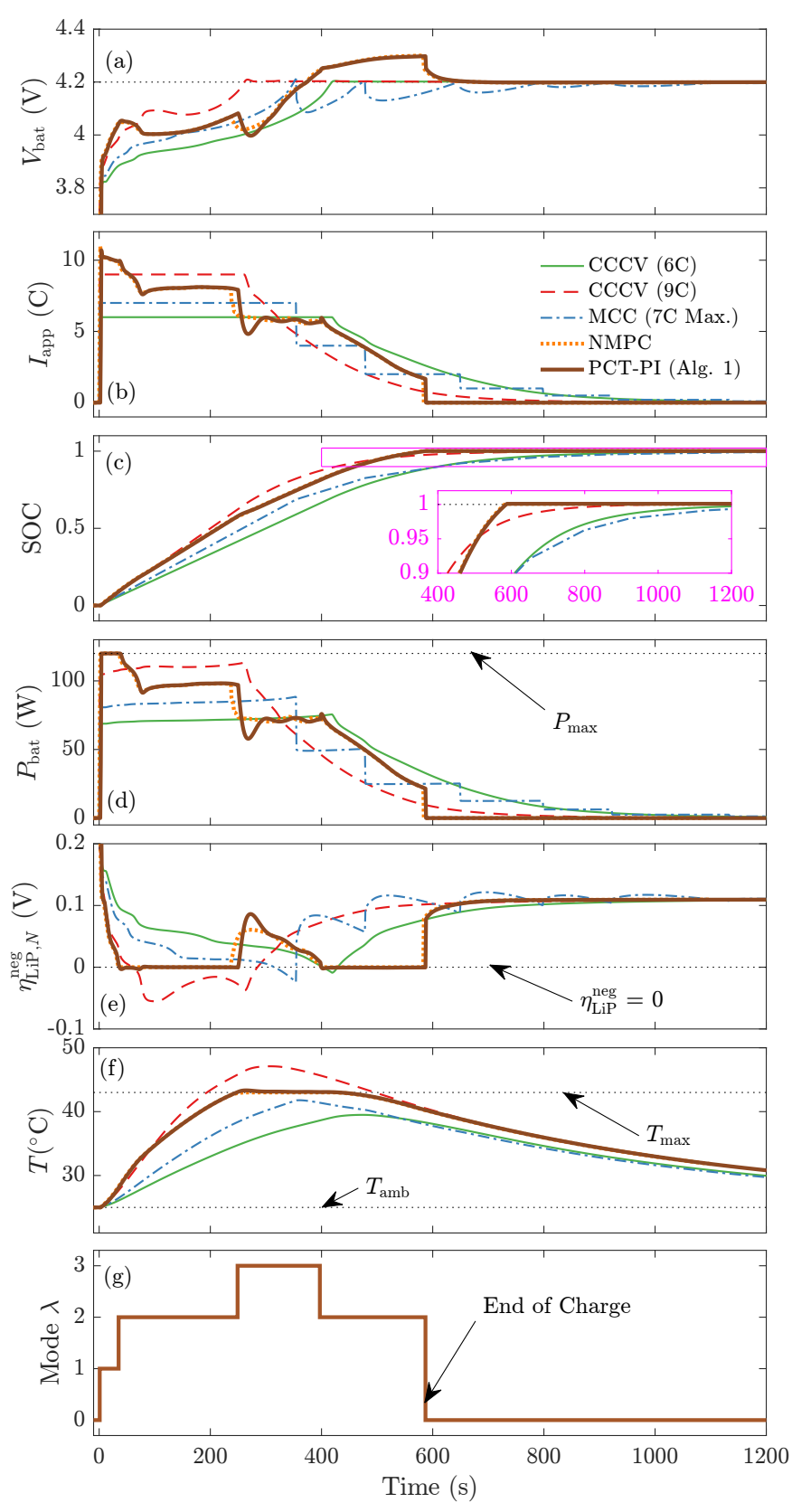

Fig. 10. Comparison of different fast charging strategies with the proposed PCT-PI strategy: (a) cell voltage, (b) current rate, (c) SOC, (d) cell power, (e) LiP potential at the neg/sep boundary, (f) cell temperature, and (g) mode of the proposed PCT-PI control. Mode 0 means no current is applied.

from Fig. 10(e) that LiP potential increases initially, while it starts to decline from about $265 \mathrm{~s}$. At $t=391 \mathrm{~s}$, the process enters Mode 2 for the second time as the LiP potential drops to zero again. The whole charging process ends in Mode 2 when the SOC reaches $99 \%$ at $584 \mathrm{~s}$.

For the model-free charging strategies, i.e., $\mathrm{CCCV}$ and MCC, it can be observed that the required charging times are much longer than the proposed strategy. Furthermore, if a high-rate current is applied for the CC stage of the CCCV strategy, it may increase lithium plating and temperature rise dramatically. For the $\mathrm{CCCV}$ with $6 \mathrm{C}$ maximum current and the MCC strategy, although the temperature is kept below its upper limit, the lithium plating cannot be fully restrained, as shown in Figs. 10(e) and (f). Hence, with the proposed fast charging strategy, the charging time is effectively shortened, and the major limiting factors such as temperature and side reaction are well controlled with the consideration of the constraints. Table III also compares the charging efficiencies of different strategies. Here the charging efficiency is defined by $\left[\int_{0}^{t_{f}} V_{\mathrm{OC}}(t) I_{\mathrm{app}}(t) d t\right] /\left[\int_{0}^{t_{f}} P_{\mathrm{bat}}(t) d t\right]$, where $t_{f}$ represents the charging time and the open-circuit voltage $V_{\mathrm{OC}}$ can be obtained as a nonlinear function of SOC. It can be seen that although not considered in the design of the present work, the charging efficiency of the proposed strategy is comparable to the CCCV and MCC strategies.

Furthermore, it can be observed that results from the proposed strategy are close to that obtained from the NMPC: The charging time of the NMPC solution is $577 \mathrm{~s}$, and the RMSE of the SOC profiles is as low as $2.7 \%$. It takes more than $1 \mathrm{~h}$ to obtain the optimal profile using NMPC even though there is no state estimation, while the proposed strategy with ETKF is considerably much more efficient (15 s). The major performance differences of the proposed strategy compared to the NMPC come from the transition stages from one mode to another: As the PI feedback control does not predict the future dynamics, there are overshoots if the constraint is expected to be well-regulated with high control gains. Nevertheless, the effects of the overshoot are insignificant, and the proposed charging control is easy to implement, thanks to its free-of-optimization strategy with a high-fidelity modelbased distributed state estimator.

TABLE III

PERformance Comparison of FAst-Charging Strategies

\begin{tabular}{cccccc}
\hline \hline & $\begin{array}{c}\text { CCCV } \\
(6 \mathrm{C})\end{array}$ & $\begin{array}{c}\text { CCCV } \\
(9 \mathrm{C})\end{array}$ & $\begin{array}{c}\text { MCC } \\
\text { (7C Max.) }\end{array}$ & NMPC & $\begin{array}{c}\text { PCT-PI } \\
(\text { Alg. 1) }\end{array}$ \\
\hline $\begin{array}{c}\text { Charging Time } \\
\left(\text { SOC }_{f}=99 \%\right)\end{array}$ & $1056 \mathrm{~s}$ & $965 \mathrm{~s}$ & $1178 \mathrm{~s}$ & $577 \mathrm{~s}$ & $584 \mathrm{~s}$ \\
$\begin{array}{c}\text { Charging Time } \\
\left(\mathrm{SOC}_{f}=95 \%\right)\end{array}$ & $708 \mathrm{~s}$ & $503 \mathrm{~s}$ & $784 \mathrm{~s}$ & $496 \mathrm{~s}$ & $501 \mathrm{~s}$ \\
$\begin{array}{c}\text { CPU Time } \\
\left(\mathrm{SOC}_{f}=99 \%\right)\end{array}$ & $3.2 \mathrm{~s}$ & $2.8 \mathrm{~s}$ & $3.3 \mathrm{~s}$ & $>1 \mathrm{~h}$ & $15 \mathrm{~s}$ \\
$\begin{array}{c}\text { Charging Effi. } \\
\left(\mathrm{SOC}_{f}=99 \%\right)\end{array}$ & 0.938 & 0.925 & 0.937 & 0.930 & 0.930 \\
\hline \hline
\end{tabular}

\section{Conclusions}

This paper has proposed a novel distributed electrochemical state estimation and fast-charging method for lithium-ion batteries using a sophisticated physics-based model. Nonuniform health- and safety-related behaviors and physical limitations across the electrodes of the lithium-ion battery were accurately predicted and taken into account for the design of a simple feedback charging control.

The following three aspects contribute to the low computational requirement for the online implementation: 1) The discretized and reformulated electrochemical battery model in the circuit form is an ODE system and thus easy to solve; 2) The ETKF is specifically suitable for the highorder stochastic battery model by only using a small sample to represent the statistical characteristics; 3) The charging 
strategy is designed using PI control laws without the need of real-time optimization.

It should be pointed out that presently, the model parameters are considered not affected by the degradation within one charging cycle. However, some of them, such as the SEI film resistance, can vary gradually as the battery ages. Therefore, online tracking of these parameters is essential and will be incorporated into the proposed fast-charging control algorithm in the future.

\section{REFERENCES}

[1] N. A. Chaturvedi, R. Klein, J. Christensen, J. Ahmed, and A. Kojic, "Algorithms for advanced battery-management systems," IEEE Control Syst. Mag., vol. 30, no. 3, pp. 49-68, Jun. 2010.

[2] A. Tomaszewska, Z. Chu, X. Feng, S. O'Kane, X. Liu, J. Chen et al., "Lithium-ion battery fast charging: A review," eTransport., vol. 1, p. 100011, Aug. 2019.

[3] M. M. Mahfouz and M. R. Iravani, "Grid-integration of battery-enabled dc fast charging station for electric vehicles," IEEE Trans. Energy Convers., vol. 35 , no. 1, pp. 375-385, Mar. 2020.

[4] P. H. L. Notten, J. H. G. Op het Veld, and J. R. G. van Beek, "Boostcharging Li-ion batteries: A challenging new charging concept," J. Power Sources, vol. 145, no. 1, pp. 89-94, Jul. 2005.

[5] A. B. Khan and W. Choi, "Optimal charge pattern for the highperformance multistage constant current charge method for the Li-ion batteries," IEEE Trans. Energy Convers., vol. 33, no. 3, pp. 1132-1140, Sep. 2018.

[6] L. Chen, S. Wu, D. Shieh, and T. Chen, "Sinusoidal-ripple-current charging strategy and optimal charging fequency study for Li-ion batteries," IEEE Trans. Ind. Electron., vol. 60, no. 1, pp. 88-97, Jan. 2013.

[7] J. Jiang, Q. Liu, C. Zhang, and W. Zhang, "Evaluation of acceptable charging current of power Li-ion batteries based on polarization characteristics," IEEE Trans. Ind. Electron., vol. 61, no. 12, pp. 6844-6851, Dec. 2014.

[8] X. Hu, H. E. Perez, and S. J. Moura, "Battery charge control with an electro-thermal-aging coupling," in Proc. ASME Dyn. Syst. Control Conf. (DSCC), no. V001T13A002, Columbus, OH, USA, 28-30 Oct. 2015.

[9] Y. Lee and S. Park, "Electrochemical state-based sinusoidal ripple current charging control," IEEE Trans. Power Electron., vol. 30, no. 8, pp. 4232-4243, Aug. 2015

[10] Z. Chen, B. Xia, C. C. Mi, and R. Xiong, "Loss-minimization-based charging strategy for lithium-ion battery," IEEE Trans. Ind. App., vol. 51, no. 5, pp. 4121-4129, Sep.-Oct. 2015.

[11] Y. Zhang, R. Xiong, H. He, and W. Shen, "A lithium-ion battery pack state of charge and state of energy estimation algorithms using a hardware-in-the-loop validation," IEEE Trans. Power Electron., vol. 32, no. 6, pp. 4421-4431, Jun. 2017.

[12] S. Wang and Y. Liu, "A PSO-based fuzzy-controlled searching for the optimal charge pattern of Li-ion batteries," IEEE Trans. Ind. Electron. vol. 62, no. 5, pp. 2983-2993, May 2015.

[13] C. Zou, X. Hu, Z. Wei, and X. Tang, "Electrothermal dynamicsconscious lithium-ion battery cell-level charging management via statemonitored predictive control," Energy, vol. 141, pp. 250-259, Dec. 2017.

[14] Y. Parvini and A. Vahidi, "Maximizing charging efficiency of lithiumion and lead-acid batteries using optimal control theory," in Proc. Amer Control Conf. (ACC), Chicago, IL, USA, 1-3 Jul. 2015, pp. 317-322.

[15] H. Rahimi-Eichi, F. Baronti, and M. Chow, "Online adaptive parameter identification and state-of-charge coestimation for lithium-polymer battery cells," IEEE Trans. Ind. Electron., vol. 61, no. 4, pp. 2053-2061, Apr. 2014.

[16] Z. Wei, J. Zhao, R. Xiong, G. Dong, J. Pou, and K. J. Tseng, "Online estimation of power capacity with noise effect attenuation for lithiumion battery," IEEE Trans. Ind. Electron., vol. 66, no. 7, pp. 5724-5735, Jul. 2019.

[17] Y. Li, M. Vilathgamuwa, T. Farrell, S. S. Choi, N. T. Tran, and J. Teague, "A physics-based distributed-parameter equivalent circuit model for lithium-ion batteries," Electrochimica Acta, vol. 299, pp. 451-469, Mar. 2019.

[18] S. Pramanik and S. Anwar, "Electrochemical model based charge optimization for lithium-ion batteries," J. Power Sources, vol. 313, pp. 164-177, May 2016.
[19] J. Liu, G. Li, and H. K. Fathy, "An extended differential flatness approach for the health-conscious nonlinear model predictive control of lithium-ion batteries," IEEE Trans. Control Syst. Technol., vol. 25, no. 5, pp. 1882-1889, Sep. 2017.

[20] R. Klein, N. A. Chaturvedi, J. Christensen, J. Ahmed, R. Findeisen, and A. Kojic, "Optimal charging strategies in lithium-ion battery," in Proc. Amer. Control Conf. (ACC), San Francisco, CA, USA, 29 Jun.-1 Jul. 2011, pp. 382-387.

[21] C. Zou, X. Hu, Z. Wei, T. Wik, and B. Egardt, "Electrochemical estimation and control for lithium-ion battery health-aware fast charging," IEEE Trans. Ind. Electron., vol. 65, no. 8, pp. 6635-6645, Aug. 2018.

[22] X.-G. Yang and C.-Y. Wang, "Understanding the trilemma of fast charging, energy density and cycle life of lithium-ion batteries," J. Power Sources, vol. 402, pp. 489-498, Oct. 2018.

[23] A. Tulsyan, Y. Tsai, R. B. Gopaluni, and R. D. Braatz, "State-of-charge estimation in lithium-ion batteries: A particle filter approach," J. Power Sources, vol. 331, pp. 208-223, Nov. 2016.

[24] J. Sturm, H. Ennifar, S. V. Erhard, A. Rheinfeld, S. Kosch, and A. Jossen, "State estimation of lithium-ion cells using a physicochemical model based extended Kalman filter," Appl. Energy, vol. 223, pp. 103-123, Aug. 2018.

[25] S. Marelli and M. Corno, "Model-based estimation of lithium concentrations and temperature in batteries using soft-constrained dual unscented Kalman filtering," IEEE Trans. Control Syst. Technol., vol. 29, no. 2, pp. 926-933, Mar. 2021.

[26] Y. Li, B. Xiong, D. M. Vilathgamuwa, Z. Wei, C. Xie, and C. Zou, "Constrained ensemble Kalman filter for distributed electrochemical state estimation of lithium-ion batteries," IEEE Trans. Ind. Inform., vol. 17 , no. 1 , pp. $240-250$, Jan. 2021.

[27] G. Evensen, "The ensemble Kalman filter for combined state and parameter estimation," IEEE Control Syst. Mag., vol. 29, no. 3, pp. 83104, Jun. 2009.

[28] C. H. Bishop, B. J. Etherton, and S. J. Majumdar, "Adaptive sampling with the ensemble transform Kalman filter. Part I: Theoretical aspects," Mon. Weather Rev., vol. 129, no. 3, pp. 420-436, Mar. 2001.

[29] N. T. Tran, M. Vilathgamuwa, T. Farrell, S. S. Choi, Y. Li, and J. Teague, "A Padé approximate model of lithium ion batteries," J. Electrochem. Soc., vol. 165, no. 7, pp. A1409-A1421, May 2018.

[30] Y. Ji, Y. Zhang, and C.-Y. Wang, "Li-ion cell operation at low temperatures," J. Electrochem. Soc., vol. 160, no. 4, pp. A636-A649, Feb. 2013.

[31] F. Zhang, C. Snyder, and J. Sun, "Impacts of initial estimate and observation availability on convective-scale data assimilation with an ensemble Kalman filter," Mon. Weather Rev., vol. 132, no. 5, pp. 12381253, May 2004.

[32] S. Moura, "Fast Doyle-Fuller-Newman (DFN) electrochemicalthermal battery model simulator," 2018. [Online]. Available: https://github.com/scott-moura/fastDFN

[33] "USABC electric vehicle battery test procedures manual. revision 2," USABC, Idaho Falls, ID, USA, Tech. Rep. DOE/ID-10479-Rev.2 ON: DE96009671, Jan. 1996.

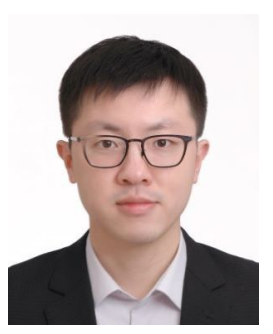

Yang Li (Member, IEEE) received the B.E. degree in electrical engineering from Wuhan University, Wuhan, China, in 2007, and the M.Sc. and Ph.D. degrees in power engineering from Nanyang Technological University (NTU), Singapore, in 2008 and 2015, respectively. From 2015 to 2018, he was a Research Fellow with the Energy Research Institute, NTU and the School of Electrical Engineering and Computer Science, Queensland University of Technology, Brisbane, QLD, Australia. Since 2019, he has been with the School of Automation, Wuhan University of Technology, Wuhan. He is currently a Researcher with the Department of Electrical Engineering, Chalmers University of Technology, Gothenburg, Sweden. His research interests include modeling and control of energy storage systems in power grid and transport sectors.

Dr. Li was a recipient of the EU Marie Skłodowska-Curie Action Individual Fellowship in 2020 


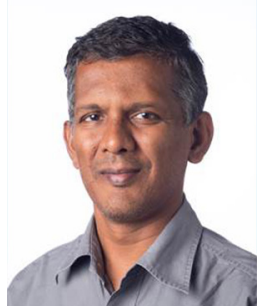

D. Mahinda Vilathgamuwa (Fellow, IEEE) received the B.Sc. degree from the University of Moratuwa, Sri Lanka, in 1985, and the Ph.D. degree from Cambridge University, Cambridge, U.K., in 1993, both in electrical engineering. In 1993, he joined the School of Electrical and Electronic Engineering, Nanyang Technological University, Singapore, where he had been a Faculty Member until 2013. He is currently a Professor of power engineering with Queensland University of Technology, Brisbane, Australia

His research interests include wireless power, battery storage, power electronic converters, electrical drives, and electromobility.

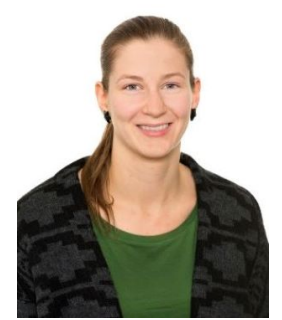

Evelina Wikner (Member, IEEE) received the B.Sc. degree in chemical engineering and engineering physics and the M.Sc. degree in nanoscience and nanotechnology from Chalmers University of Technology, Gothenburg, Sweden. She is a postdoctoral researcher with the Department of Electrical Engineering, Chalmers University of Technology, where she also earned her Ph.D. degree in 2019.

Her research interest is in Li-ion batteries, optimizing utilization and lifetime in stationary and automotive applications. Her main area of expertise is in testing and physics-based modeling of Li-ion batteries. In her current research project, different automotive driver behaviors are analyzed and how the different driver behaviors affect the battery aging is investigated through testing and physics-based modeling.

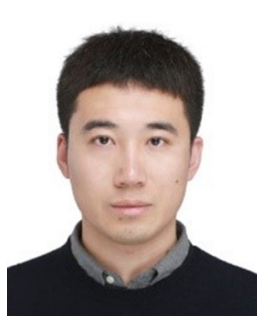

Zhongbao Wei (Member, IEEE) received the B.Eng. and M.Sc. degrees in instrumental science and technology from Beihang University, Beijing, China, in 2010 and 2013, respectively, and the Ph.D. degree in power engineering from Nanyang Technological University, Singapore, in 2017. He was a Research Fellow with the Energy Research Institute, Nanyang Technological University, from 2016 to 2018 . He is currently a Professor of vehicle engineering with $\mathrm{Na}-$ tional Engineering Laboratory for Electric Vehicles, School of Mechanical Engineering, Beijing Institute of Technology, Beijing. He has authored more than 40 peer-reviewed articles. His research interests include battery modeling, identification, state estimation, diagnostic, and thermal management, with applications to renewable energy systems such as lithium-ion battery and vanadium redox flow battery.

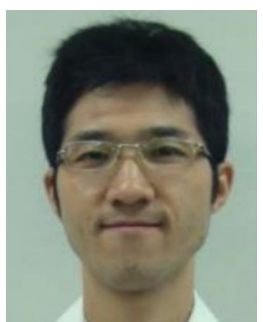

Xinan Zhang (Member, IEEE) received the B.E. degree in electrical engineering and automation from Fudan University, China, in 2008. He received the $\mathrm{Ph} . \mathrm{D}$. degree from Nanyang Technological University (NTU), Singapore, in 2014. Then, he worked as a postdoc researcher in NTU and the University of New South Wales from 2014 to 2017. He worked as a Lecturer in NTU from June 2017 to September 2019. In September 2019, he joined the University of Western Australia as a Senior Lecturer. His research interests include electrical machine drives, control and modulation of power electronic converters and management of hybrid energy storage systems.

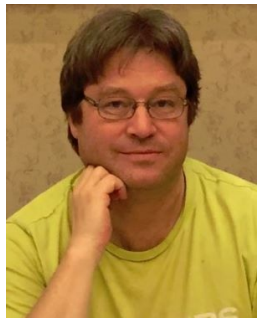

Torbjörn Thiringer (Senior Member, IEEE) works at Chalmers university of Technology, Gothenborg, Sweden, as a professor in applied power electronics. He took his M.Sc. and Ph.D. degrees at Chalmers University of technology in 1989 and 1996 respectively. His areas of interest include the modeling, control and grid integration of wind energy converters into power grids, battery technology from detailed cell modelling to system aspects, as well as power electronics and drives for other types of and industrial applications. applications, such as electrified vehicles, buildings

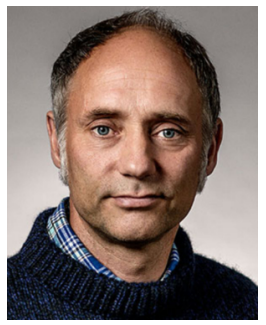

Torsten Wik received the M.Sc. degree in chemical engineering (major in applied mathematics), the Licentiate of Engineering degree in control engineering, the Ph.D. degree in environmental sciences (major in automatic control), and the Docent degree in electrical engineering from the Chalmers University of Technology, Gothenburg, Sweden, in 1994, 1996, 1999, and 2004, respectively. From 2005 to 2007 , he was a Senior Researcher with Volvo Technology, Gothenburg, researching on control system design for combustion engine test cells, and combined reformer and fuel cells. He is a Professor and the Head of the Automatic Control Group, Department of Electrical Engineering, Chalmers University of Technology.

His current research interests include optimal control, model reduction, and systems with model uncertainties, with applications to energy storage, environmental, and biological systems.

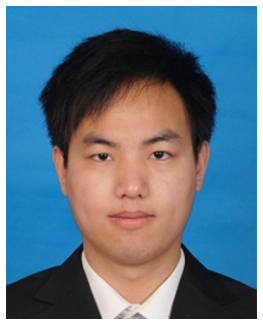

Changfu Zou (Member, IEEE) received the Ph.D. degree in automation and control engineering from the Department of Mechanical Engineering, University of Melbourne, VIC, Australia, in 2017. He was a Postdoctoral Researcher with the Chalmers University of Technology, Gothenburg, Sweden, where he is currently an Assistant Professor with the Automatic Control Group, Department of Electrical Engineering.

His current research interests include modeling and control of energy storage systems for automotive and power grid applications

Dr. Zou was a recipient of the Swedish Research Council Starting Grant, Marie Skłodowska-Curie Fellowship, and a number of grants funded by, e.g., the Swedish Energy Agency and Innovation Agency. He serves as an associate editor for IEEE Transactions on Transportation Electrification. 